\title{
Situación del Grupo Andino en el contexto latinoamericano e internacional
}

VALIDEZ DE LA INTEGRACYÓN ANDINA EN EL CONTENTO ACTUAL LATINOAMERICANO Y MUNDIAL

El tema que se me ha solicitado, versa sobre la situación del Grupo Andino en el contexto internacional y latinoamericano. Tratar un tema de esta naturaleza implica plantearse una primera $y$ básica pregunta: ¿Tienen aún validez las razones por las cuales se creó el Grupo Andino y responde éstc a los desafíos que los paises que lo conforman tienen que enfrentar en el último cuarto del siglo xx? En otras palabras, los diagnósticos, estudios y realizaciones logradas en estos últimos años, ¿son base suficiente para abocarse a la conquista de nuevos horizontes $y$ de nucras frontemas que hagan más viable el desarrollo de los países andinos?

Estas preguntas son profundamente relevantes y de alta significación en la situación actual de las relaciones internacionales.

Después de once años de la Declaración de Bogotá y de ocho años de la firma del Acuerclo de Cartagena, es necesario detenerse un momento para poder analizar con profundidad algunos de estos interogantes. Quisiera comenzar a responder estas preguntas recordando las palabras del presidente del Comité de la Comunidad Económica Europea, señor Jenkins, quier dijo en un discurso pronunciado recientemente: "Siempre basé mi convicción de ciudadano curopeo en la certidumbre intelectual de que raros son los problemas que se pueden resolver en el plano puramente nacional".

Estos conceptos, dichos por un ciudadano británico después de casi treinta años de existencia del Tratado de Roma, son profundamente inspiradores y aleccionadores para nosotros los ciudadanos de esta comunidad andina.

La pregunta sobre la valícle\% del Gupo Anclino en el contexto actual de la situación mundial y latinoamérica, sólo tiene una respues. ta: el Grupo Andino sigue y seguirí teniendo un profundo signilicado para un clesarrollo más pleno de nuestros pueblos y para una mejor y más independiente inserción económica y política en el contexto latinoamericano e internacional, porque es verdad ayer como 
hoy, especialmente para nuestros países, que "son raros los problemas que se pueden resolver en el plano puramente nacional".

¿Es sólo voluntarismo el afirmar en forma tan categórica to anteriormente dicho? Los diagnósticos que hicimos en los años sesenta, a los cuales tanto contribuyera la CEPAL, siguen siendo válidos en sus líneas más fundamentales. No es viable para países medianos y pequeños lograr un desarrollo económico y social acelerado y autosostenido en el marco estrecho de economias nacionales y de hipertrofia de soberanias políticas, sobre todo pensando en la situación latinoamericana y mundial a fines de este siglo.

Es conocido por todos ustedes que hasta los años sesenta, los países latinoamericanos en general, tuvieron un modelo de desarrollo el cual contenia dos procesos paralelos. Por un lado, abiertos al mercado internacional, especialmente a través de sus exportaciones de materias primas y, por el otro, cerrados hacia adentro en un esquema de sustitución nacional de importaciones, con altos niveles de protección $y$, por lo tanto, con grados elevados de ineficiencia.

Este modelo de desarrollo, posterior a la gran crisis y a la Segunda Guerra Mundial, estuvo centrado en esta doble racionalidad, que era compatible con la división internacional del trabajo que surgió en esos años y con el orden económico mundial que consolidó dicha división, especialmente por la influencia y el pođer que ejercían los países del centro.

Los países latinoamericanos, a pesar de la cadencia de sus reuniones tan retóricas y de acuerdos jurídicos superestructurales, vivieron dándose las espaldas y manteniendo cada cual sus propias estrategias de desarrollo que, por ser aisladas, eran en la mayoria de los casos ineficientes, y consolidando un alto grado de dependencias de los países industrializados. Hay que recordar que en el momento de firmarse el Tratado de Montevideo, aunque éste sea un índice superficial, el comercio intra-regional alcanzaba la suma de US\$684 millones y el intra-subregional andino no llegaba a los 50 millones de dólares.

Sin embargo, al final de los años 50 y el comienzo de la década de los 60, se fue generalizando en la región la conciencia de que el modelo de desarrollo latinoamericano de postguerra entraba en crisis de agotamiento. En otras palabras, era necesario buscar nuevas estrategias de desarrollo para una nueva forma de inserción en el mundo internacional. De allí fueron surgiendo los diferentes procesos de integración regional $y$ subregional con sus múltiples formas y experiencias.

El gran aporte conceptual que surgió en la década pasada a nivel latinoamericano fue la generación de esta conciencia politica de que 
toclos los paises necesitaban de la cooperación e interdependencia económica y política, obviamente con grados cliferentes para países de diferentes tamaños, si querian aumentar sus ritmos y mejorar sus estilos de desarrollo, especialmente en el sector industrial y generar un nuevo poder negociaclor con el mundo cxterior.

Quiero sólo recordar en esta ocasión que esta creciente conciencia política Ilevó a que en 1967 todos los Presidentes de las Américas proclamaran en forma unánime, en Punta del Este, el acuerdo de crear progresivamente a partir de 1970, en un plazo de quince años, el Mercado Común Latinoamericano. El viejo anhelo de una América Latina unica alcanzaba en esa ocasión su más alto reconocimiento politico y parecía existir el consenso y la voluntad suficiente para comenzar a hacerlo realidad. Es interesante recordar que por primera vez, el Gobierno de Estados Unidos, declaraba que la Integración Latinoamericana era un proceso que formaba parte de su política hemisférica. Desgraciadamente, tan ambiciosa como amplia concepción "que llevaba en sí arcluos problemas prácticos a resolver" quedó al poco tiempo detenida $y$ ' como acuerdo real de todo ese gran movimiento, sólo quedó reconocida la persistente voluntad de los paises andinos de realizar entre ellos un esfuerzo especial y orgánico de integración, en el expreso entendido de que esa nueva unidad significaba un paso necesario para un entendimiento más armonioso con los paises més grandes del contincnte y no una separación con éstos.

In este contexto surgió el Grupo Andino. Es necesario recordar aquí algunas de las caracteristicas polílicas que rodenron su formación. La primera de ellas fue la relativa homogeneidad de la orientación económica y política de los gobiemos de ese entonces. Sólo baste recordar algunas frases de la Declaración de Bogotá. En ella se dice, por ejemplo: "Abrigamos la convicción de que el afianzamiento de las instituciones propias de ia democracia representativa, mediante la activa intervención del pucblo, y la participacjón creciente de éste en los benelicios del progreso, el respeto a los clerechos humanos $y$ el desarrollo económico y social, son condiciones esenciales e inseparables para canalizar la libertad y el bienestar de las naciones americanas". Més adelánte, la misma Declaración scr̃alaba: "La necesidad de realizar reformas sustanciales en las estructuras institucionales, económicas $y^{\prime}$ sociales que permitan satisfacer las justas cxigencias do nuestros pueblos".

No fue sólo entonces la claridad de un diagnóstico en relación a las limitaciones que dentro del marco de la ALALC tenían los países de mercaclos insuficientes o de menor desarrollo relativo, lo que impulsó a la creación del Grupo Andino. Fue, junto a lo anterior, una 
común visión política que posibilitó y facilitó todo el proceso de ncgociaciones que condujo en mayo de 1969 a la firma del Acuerdo de Cartagena.

Contestando la pregunta inicial sobre la vaide\% del Grupo Andino, me parece necesario señalar algunos hechos que continúin sicndo relevantes a pesar de los cambios ocurridos en el interior de los países, en América Latina y en el munclo, a los cuales nos referiremos más adclante.

En primer término, considero que sigue sienclo válida la afirmatción que fuera estipulacla on la Declaración de Bogotá, y en los Acuerdos de la Reunión de Presidentes de Punta del Este, en el senticlo de que para hacer posible la integración latinoamericana, son necesariats la existencia, clesarrollo y éxito de esquemas subregionales de integanción econónica y social, puesto que parece evidente que la inregración lálinomericana no se logrará, como se pensó a fín'es de la década de los 50 , por un gytan acuerclo que fije un marco único a todos los patses de la región individunlmente consiclerados. Pienso que ella será posible sólo si se logra desarrollar lo que alguien llamara "centros de integmación", entre los cuales se estructuren formas dinámicas de relación y cooperación por acuerdos políticos o por seclores industriales, de servicios o tecnológicos. La di Lerencia en los niveles de desárrollo y de recursos y espacios económicos, demostró ser una valla fornidable para la concepción de una clesgravación interna mecínica alutomátical, en circunstancias que las corrientes comerciales per se no eran ni suficientes ni equitativamente distribuidis.

Tiene también actualiclad la afirmación cle que la ALALC era y es un instrumento limitaclo y que, especialmente, no satisfacia los intereses de los paises cle mercados insuficientes y de menor desarrollo econónico relativo.

Como hace algunos años, sigue siendo una evidencia, aunque algunos quieran negarlo, que para la gran mayoría de estos paises, entre un esquema de sustitución macional de importaciones y un modelo de clesarrollo abierto a la compecencia mundial, hay un camino Iógico, aclecuado a nuestras posibiliclades, difícil pero indispensable, cual es la creación de un espacio económico capaz cle dar cabida a producciones eficientes, a precios competitivos, a economins de escala y a una especialización industrial. Si ese fue cl camino seguido por Estaclos Uniclos en su época de lomación, ha siclo el de la Comunidad litropea y del CANEE, es el que siguen los paises ámbes y en gran medicla los escandinavos, no escapa a la lógica su natural seguimiento por países que han iniciado tardiamente su industrialización y luchan por nuevos niveles de independencin. 
Los ejemplos mundiales y la necesidad histórica actual exigen organizar los vecindarios en unidades orgánicas al servicio de los pueblos, formar grupos o vínculos especiales entre países que se complementan. Salir alguna vez del siglo xux y prepararse para el XXI que se asoma.

La experiencia mundial y la de algunos paises mayores en la región muestra la indispensable necesidad de mercados ampliados para desarrollar industrias y servicios que puedan lograr incorporarse a la economía internacional en términos competitivos. Los casos de Brasil, Argentina y México son ejemplos fehacientes. En una medida decisiva su gran mercado interno es el que ha posibilitado que las exportaciones manufactureras brasileras, por ejemplo, pasasen de 869 millones de dólares en 1972 a 2.500 millones de dolares en 1975 , llegando a representar alrededor del $30 \%$ de las exportaciones tota les de ese pais y aumentando simultáneamente su consumo interno. En el caso argentino, ha habido una tendencia similar llegando en 1974 sus exportaciones manufactureras a los 1.200 millones de dólares. Para este pais, el mercado regional ha sido de una importancia fundamental en el desarrollo de sus exportaciones industriales.

El propio Grupo Andino es una demostración concreta de la significación del mercado ampliado, especialmente para el sector industrial. Baste solo recordar que en 1969 el comercio intra-subregional no alcanzó a los 170 millones de dólares llegando, según cîfras provisionales de la Junta del Acuerdo de Cartagena, a los 876 millones de dólares en el año recién pasado. Estudios de este organismo muestran que en el Grupo Andino se comercializaba ya alrededor del $30 \%$ de todas las exportaciones manufactureras de los países que lo formaban. Todo lo anterior muestra que sigue teniendo validez la idea de que el mercado ampliado es un elemento esencial para el aumento del potencial exportador y para un racional desarrollo industrial de estos paises pequeños y medianos.

Sigue también en pie la significación que se dio al negociar el Acuerdo de Cartagena a la programación industrial como un elemento fundamental del proceso, para no dejar todo el universo arancelario al mecanismo de liberación del comercio. Era a través de la programación conjunta que se pensaba racionalizar la producción de los sectores más dinámicos y orientar, por lo tanto, el proceso de asignación de recursos a nivel subregional, de modo tal que de él se obtuviera una alta eficiencia dentro de un marco de especialización de la estructura productiva y una corrección dinámica a los desniveles existentes. Fs este el elemento más interesante y novedoso del Acuerdo, pues pone a prueba la disciplina de los paises miembros en la consecución comunitaria del desarrollo. Es esta disciplina la 
que genera la solidaridad, como se han visto ejemplos notables en Europa, a causa de los fuertes desequilibrios económicos entre los países miembros de la Comunidad.

A este respecto, cabe señalar la preocupacion que surge del hecho de que se haya cumplido el plazo de la programación industrial con la sola aprobación de dos programas sectoriales de desarrollo, teniendo presente las dificultades inherentes a un esfuerzo tan complejo pero, al mismo tiempo, tan apasionante como es la programación conjunta de las más importantes ramas industriales. Esperamos conliados que en cl nuevo plazo adicional de dos años, el Grupo Andino se consolide en forma definitiva, muy especialmente aprobando las propuestas sobre nuevos programas sectoriales de desartollo industrial intensificando la puesta en march del mecanismo de la liberación del comercio y asegurando el equilibrio en los resultados de cste proceso. Este hecho tendria una alta significación no sólo para el Grupo Andino, sino para las posibles relaciones entre este Grupo y los denás países latinoamericanos, especialmente Argentina, Brasil y México.

Señalamos también al iniciar el Acuerdo, que uno de los elementos más fundamentales para un desarrollo más autónomo de estos paises, era tener una base suficiente para el progreso de la investigación científica y del desarrollo tecnológico apropiado a los requerimientos de estos países. La experiencia del Grupo Andino en este sentido es también una confirmación de la validez de nuestro diagnóstico y una lección para tuuchos paises en desarrollo. Los programas andinos de desarrollo tecnológico en la hidro-metalurgia del cobre, en el bosque tropical y en otros campos, muestran que el espacio ampliado también tiene su significación para el uso de las economías de escala que requĩeren la investigación cientifica y el desarrollo o adopción de tecnologías.

Ya en la Declaración de Bogotá se hizo mención a la necesidad de mantener una posición común frente al capital extranjero. La Decision 24 ha demostrado a América Latina y al mundo en desarrollo que un grupo de países con voluntad política puede tener capacidad negociadora y ofrecer al capital extranjero condiciones de estabilidad pero, al mismo tiempo, de justicia y de dignidad en la relación entre un estado o inversionistas extranjeros. En su momento habrá que analizar los sostenidos esfuerzos para desacreditar esta decision, tan obvia como lúcida, provocados por parte de sectores internacionales interesados en mantener su dominio en los mercados latinoamericanos, invocando en su favor la libertad económica como el dogma del progreso. Entre desiguales, la libertad siempre ha conducido a la injusticia cuando no al abuso sobre el más débil. Sólo 
una voluntad politica basada en las experiencias del pasado y alimentada por una concepción de un futuro más autónomo, puede asegurar el equilibrio de lo que es útil para las partes. En la difícil lucha por el desarrollo, tanto en el plano interno como en el externo, la aceptación fácil de inversiones externas o la satisfacción de necesidades no esenciales generalmente se pagan con mayores dependencias o distorsiones económicas y sociales.

Es necesario señalar también la importancia que el Grupo Andino tiene para América Latina, si logra clefinitivamente consolidarse en un futuro cercano. El puede ofrecer una equilibrada capacidad de negociación con los países mayores de la región y con los otros subsistemas de integración, de tal modo que en el marco de SELA la región pueda estructurar un complejo de formas de cooperación que le den pragmáticamente una solidaridad interna $y$, al mismo tiempo, una personalidad y un poder frente al mundo internacional. Desde su concepción, el Pacto Andino se percibió como el esfuerzo acelerado de un grupo de países para integrarse, como unidad, en. un concierto más armónico de políticas y comercio con los países mayores de la región.

Quisiera detenerme sobre esto y profundizar en algunos conceptos. En primer lugar, estoy convencido que, en gran medida, sin Grupo Andino no habrá integración latinoamericana. En segundo término, sin integración, la distancia relativa de los países de la región entre sí aumentará. Si se comparan las cifras económicas de los países miembros de la ALALC de 1969 y la de los mismos países hoy -con excepción de Venezuela, por razones conocidas- se puede apreciar un distanciamien to muy marcado y creciente que favorece a los países más grandes. Es la integración la que permitiría que muchos problemas históricos que aún dividen, puedan encontrar un cauce nuevo y dinámico que haga viables soluciones prácticas, propias a los tiempos que se viven que son de complementaridad en la construcción de vecindarios orgánicamente unidos y no de naciones aisladas satélites de los grandes centros. Por último, sin integración no será posible una forma de inserción beneficiosa de los países de la región en el concierto mundial para crear un orden internacional justo. Esto es válido no sólo para los pequeños países, sino también para los más grandes y tal vez puede llegar a ser la razón más po. derosa. 
EL FUTURO DE LA COOPERACIÓN REGIONAL

Las afirmaciones anteriores no pueden interpretarse como un optimismo desmedido, aunque yo sea un integracionista cada vez más convencido e irreductible. Tal vez influya en esta convicción ahondada la angustia con que veo - -después de seis años de vivir cerca de los grandes centros - a nuestra América desdibujarse en el concierto mundial, perder sonoridad e identidad, a veces desafinar y ser un ramal y no un centro por donde pasa el curso de los que deciden la historia, determinan los estilos de vida y construyen el futuro.

Quisiera ser muy claro y directo para afirmar que veo con preocupación el futuro no sólo de la integración andina sino de todo el proceso de cooperación económica y política regional.

Los tiempos están cambiando en el mundo y en América Latina en una forma rápida y radical. Ello obliga a detenerse y a reflexionar con mayor profundidad sobre nuestra experiencia pasada y también sobre las grandes transformaciones ocurridas al interior de nuestros países, en la región como un todo y en el mundo. Debemos de esta manera, extraer las lecciones de nuestra historia próxima y discernir las alternativas que llevan con realismo hacia la consolidación de la solidaridad política y' económica de la región y a su relación e inserción en el nuevo orden mundial. Especialmente deb'emos ser lúcidos para descubrir las exigencias que el futuro del mundo y de la región al final de este siglo nos imponen para el quehacer de hoy.

Sería simplista creer que el movimiento de cooperación regional generado en la década pasada se pueda proyectar linealmente para los años que vienen. Y que sólo estamos enfrentados a una crisis de crecimiento en el proceso de integración. Los problemas que este proceso presenta, a pesar de los avances alcanzados, tienen múltiples causas, muchas de ellas independientes pero, singularmente, ti'enen una estrecha relación con los problemas políticos, sociales y económicos de nuestros propios países.

Quisiera en esta ocasión adelantar una hipótesis a este respecto. Creo que en nuestra América Latina de hoy debemos, como nunca, tratar de presentar para el futuro la solidaridad básica indispensable con la cual en el pasado se dieron pasos tan importantes como la formación del Grupo Andino, se hizo la Reunión de Presidentes de Punta del Este, se logró el consenso de Viña del Mar y se adoptaron posiciones comunes en diversos foros y reuniones internacionales. Hoy 
es necesario admitir con realidad que las relaciones y la solidaridad intrarregional, están teniendo graves obstáculos y dificultades.

Nuestros países, al despertar ciel sueño de un interamericanismo igualitario en lo juridico y crecientemente desigual en todos los demás factores que constituyen la médula del desarrollo; al comenzar a tomar conciencia de la necesidad de una posición común latinoamericana, hacia adentro y hacia afuera que encontró uno de sus mejores momentos en el consenso de Viña del Mar, se vieron abocados a definir sus intereses comunes, su acción conjunta en la región y frente al mundo. Durante estos últimos años se fue aceptando, con razón, la noción del pluralismo en las relaciones intralatinoamericanas. Nos dimos cuenta que era posible estructurar un marco de acción regional y sub-regional conjunta aceptando la diversidad de sistemas politicos, económicos $y$ 'sociales.

Pero, emergieron en la región nuevos problemas y realidades fruto de la movilización social y política de las grandes mayorias y de las dificultades que los sistemas institucionales de algunos paises y los ritmos de desarrollo posibilitaban para responder con eficiencia a las operaciones populares. Profunda gravitación ha tenido en este proceso el dinamismo de un sistema económico internacional, organizado para el propio beneficio de los centros, que ha funcionado implacablemente, en periodos de progreso como en el de recesión.

De todo lo anterior no podían escaparse los procesos de integración. Ellos sufricron también las consecuencias, no del pluralismo, sino de distanciamientos en las relaciones entre importantes países latinoamericanos. En la base de estos distanciamientos está el haber Ilegado a diferencias aparentemerte críticas en la concepción de los modelos de desarrollo, de los sistemas políticos, en la existencia de orientaciones ideológicas contrapuestas, y de diferentes posiciones frente a la realidad económica, politica e ideológica mundial. Es decir, gradualmente se pasó de la homogeneidad básica a la aceptación del pluralismo en las relaciones hemisféricas y de allí al distanciamiento. En alguna medida teremos que aceptar que hemos desandado el camino recorrido y que de alguna manera hemos vuelto a un pasado, no de autonomía para crecer hacia adentro sino de autonomía para buscar cada cuali su propia vinculación o inserción en las economías desarrolladas de los centros. La integración no ha alcanzado a ser un instrumento capaz de impedir la vulnerabilidad y la dependencia externa que caracterizan a nuestra región más que a ninguna otra.

Junto a lo anterior, algunos países con grandes dimensiones económicas y humanas y con estabilidad interna, han dado significativos pasos que, en importante medida, los han distanciado del resto 
de los países medianos y pequex̃os, algunos de los cuales se mantienen aún en niveles de desarrollo muy poco superiores a los de hace diez o quince años. Es decir, proyectando el futuro, en el continente se están dando las condiciones de una tendencia a reproducir entre países de la región, las situaciones de marginalización que hoy denunciamos en el mundo en su conjunto. Por ello, la problemática de los países de mercado insuficiente y de menor desarrollo relativo sigue siendo un factor central en todo esquema de cooperación regional o sub-regional que el Grupo Andino supo prever con claridad y decisión. Esta consideración no implica, de manera alguna, una crítica a los países mayores de la región, cuyo crecimiento, como latinoamericanos, debemos celebrar. Es la comprobación simple de una realidad.

Más aún, viejos problemas no han sido resueltos. Siguen siendo un desafío intelectual y político para nuestros países. Me refiero, por ejemplo, a problemas derivados del marcado dualismo que se está creando entre sectores dinámicos de alta concentración de progreso técnico, de los frutos de la educación, de la participación política y social por un lado y la presencia de grandes sectores proletarios marginados, los cuales no tienen oportunidades mínimas para ser sujetos y actores políticos, económicos y sociales. La integración $y$; en particular, el Pacto Andino, no han sido concebidos para extender una red de privilegios y hacer comunitaria la pobreza. Por el contrario, es! aquí donde, tal ve $z$ el Grupo Andino puede encontrar bases de apoyo sociales que están latentes pues son los pueblos los que guardan, con mayor generosidad, el espiritu latinoamericano junto con sus valores culturales profundos.

Otro de los problemas que no puede eludirse, es el de la redistribución del ingreso, diagnosticado hace ya muchos años. Floy tenemos que aceptar con preocupación que en América Latina hay 100 millones de pobres, lo que tendrá tarde o temprano, una enorme relevancia en las proyecciones políticas y sociales de nuestros países. Una política de empleo está íntimamente unida a una programación conjunta de recursos financieros y tecnológicos y ambas deben ser consideradas como una responsabilidad solidaria pues el empleo llegará a ser el problema más acuciante cle América Latina.

Por fin, quiero señalar que el antiguo problema de la vulnerabilidad externa sigue teniendo una especial relevancia para algunos países de la región. Salvo excepciones, no hemos podido lograr mecanismos apropiados para evitar la propensión a la inestabilidad de los precios, al desequilibrio en las balanzas de pago y a la inflación, sumando a lo anterior el elevadísimo nivel de endeudamiento externo que la región presenta. La apertura hacia el exterior ha 
llevado a América Latina a un endeudamiento externo -público y privado- de más de 65 millones de dólares para los países no exportadores de petróleo con. un pago por tecnología importado que llega a 2.000 millones y que aumenta a razón de $18 \%$ al año. Por su parte, el déficit en cuenta corriente en los mismos países en su balanza de pagos llegó a ser igual a la mitad del valor de sus exportaciones en 1975 .

Todos estos son factores internos y externos que parecen conspirar en contra de la integración. Ciertamente no son causados por lo que en ese campo se haya hecho. A mi juicio, por el contratio, corresponden en una medida decisiva a los estilos de desarrollo seguidos a nivel nacional que sufren de las deformaciones propias a una deficiente y pasiva inserción en la economía mundial. La integración no es un remedio universal, no es una panacea, pero es el lógico camino que ya Benjamín Franklin indicó: "Unidos nos defenderemos, divididos seremos destruidos". El consejo, fielmente seguido, creó la nación más poderosa de la tierra.

Es por este conjunto de razones que cada vez con más convicción pienso en Ia importancia que puede tener un Grupo Andino consoliclado, como instrumento para enfrentar este tipo de problemas $y$, además, para crear las condiciones de un acercamiento político en las relaciones intralatinoamericanas. En este sentido, este Grupo es un centro vivo, único, de conciencia regional, de posibilidades prácticas para crear en Brasil, México y Argentina, y otros paises, una soliclaridad latinoamericana de un nivel más dinámico, $y$ una abierta y creadora relación con las otras experiencias de integración sub-regional. Tal rez, nunca antes se vio como ahora la necesidad de sostener y hacer avanzar este esfuerzo, abierto hacia los paises que pueden a él incorporarse, abierto con identidad a otros paises de la región, con una visión de una arquitectura económica que utilice los materiales modernos que otros continentes, con audacia, están empleando particularmente en empresas conjuntas de financiación. y de tecnología.

$\mathrm{Si}$ a ello se agrega la existencia del SELA, instrumento reciente pero fundamental para la región, al interior del cual los diversos intereses se vayan encontrando en múltiples formas y en una diversidad de relaciones, programas y proyectos, se puede tener optimismo de que América Latina puede hacer converger sus intereses y no ahondar sus diferencias, sino más bien caminar más maduramente $\mathrm{y}$ con paso más seguro hacia la integración regional. 


\author{
EL PROCESO DE INTEGRACIÓN REGIONAL Y SUBREGYONAL \\ EN EL CONTEXTO MUNDHL
}

Permitaseme ahora plantear una segunda pregunta, para mí fundamental. ¿De qué manera influye la actual situación internacional sobre el proceso de integración andina? En otras palabras, la crisis económica y política internacional y la quiebra del viejo orden mundial, ¿debilitan la integración subregional o, por el contrario, son un elemento dialéctico que urge a los países a dar nuevos pasos que aumenten y estrechen su interdependencia?

Para contestar esta pregunta debemos tener claridad sobre las características fundamentales de la actual situación mundial y de sus principales tendencias futuras.

De todos es conocida la crisis manifiesta del antiguo orden económico mundial y de la división internacional del trabajo que la sustentaba. Esta crisis tiene repercusión no sólo en' el orden económico sino también en el orden político, social y cultural. Sus efectos, como es natural, caen con todo su peso en el mundo en desarrollo.

Sin duda alguna, en las últimas décadas ha hapido un aspecto positivo que es necesario señalar y es el de la distensión. El fin de la guerra fria y la rigidez de la estructura de bloques, es decir del mundo bipolar, es un logro de la humanidad. Pero la distensión de por sí, no lleva implícita la génesis de una nueva estructura de las relaciones mundiales, pero ha sido, por lo menos, una ventana abierta para que se pueda expresar el conjunto de latentes tensiones que la guerra fría había reprimido pero que subterráneamente existían.

Más allá de ciertos efectos que han hecho más evidente y manifiesta la crisis actual, como son los problemas monetarios, los problemas de la inflación mundial, etc., la distensión ha hecho aparecer causas más profundas que son las características más relevantes de lo que hoy día vive la humanidad.

En primer término, ha quedado de manifiesto que el tipo de crecimiento de los pafses del centro era sólo posible bajo el esquema del anterior orden internacional y de la anterior división del trabajo. Este tipo de crecimiento estaba fundamentalmente basado en el suministro constante de materias primas baratas y de una relación en los tèrminos del intercambio extremadamente favorable para los países industrializados. Al aumentar el control y al hacer efectivo el poder de negociación de los países en desarrollo sobre algunas de sus 
materias primas, especialmente aquellas más escasas, los países industrializados han descubierto con más claridad cuál es la debilidad central de su tipo de desarrollo. Si a esto se agrega la mayor conciencia de las limitaciones ecológicas que existen en los países industriales pada mantener un. crecimiento ilimitado, la escasez futura de recursos naturales no renovables y el surgimiento del desarrollo industrial en los paises de la periferia, es evidente que el modelo de desarrollo en los países del centro deberá variar fundamentalmente, poniéndose más énfasis en un crecimiento más bien cualitativo que cuantitativo, Es lo que los países más civilizados comienzan a plantear como la nueva etapa de su ćesarrollo nacional, que tendrá un profundo impacto no sólo en la economía de los países en desarrollo que han seguido y que internacionalmente serán denunciados como obsoletos e injustos.

Un segundo elemento de la actual situación mundial que ha emergido es la entrada en escena del llamado Tercer Mundo. Con todas sus limitaciones y sus diferencias internas, lo que podríamos llamar "la movilización de las mayorías" hoy día es una realidad, no sólo en el plano nacional sino también en el plano internacional. Sus componentes están buscando con mayor fuerza que nunca mejorar su participación en los beneficios del progreso, reclamando para ello relaciones de justicia internacional dentro de un nuevo orden mundial y, al mismo tiempo, creando lazos de solidaridad entre países en desarrollo que amplien sus posibilidades de crecimiento económico y de negociación con el resto del mundo. En este sentido, el primer avance lo han dado los paises productores de hidrocarburos, pero se dan otros pasos que hacen avanzar otros frentes que se seguirản dando en el futuro, por parte de otras asociaciones de productores de materias primas. La solidaridad entre ellas será un elemento clave en el poder que puede ejercerse en beneficio de la gran mayo. ría de los países en desarrollo, especialmente del denominado Cuarto Mundo.

En tercer lugar, caracterizan a la coyuntura mundial graves problemas de orden financiero que han surgido en los últimos años y que han producido la llamada stag-flation. No vale la pena repetir que el sistema monetario internacional surgido de los acuerdos de Breton Wood ya no funciona, pero todavía no se logra un nuevo esquema que haga compatible los intereses de los paises desarrollados y en vías de desarrollo. Es necesario aquí subrayar que uno de los mayores problemas futuros está en el monto y términos del endeudamiento externo que estos últimos patses tienen y que necesariamente hará crisis si no se busca nuevas formas de transferencia de recursos financieros a los países en desarrollo. Quiero también seña- 
lar que en el mundo desarrollado se abre camino la necesidad de buscar, para ese mundo, una sanidad económica y financiera, que está intimamente vinculada a cambiar los objetivos de un crecimiento económico basadas en la irracional producción de lo superfluo y en el insensato gasto militar.

La cuarta característica es la crisis de la cooperación internacional en la cual América Latina quizás sea la región más dañada. Lo indica, entre otros indices, la disminución de la ayuda oficial y el aumento de los préstamos privados. Es de todos conocido que los países desarrollados, salvo excepciones, no han logrado su compromiso de dedicar el $1 \%$ de su producto geográfico bruto a la cooperación intemacional. Pero no sólo es el problema del monto el que está en juego, sino también el de las modalidades en que se otorga esta cooperación, no solamente en cuanto a los préstamos oficiales sino, muy especialmente, en el comportamiento de algunos estados y corporaciones multinacionales, que no han respetado la soberanía ni la idignidad de los pueblos en desarrollo. Es importante subrayar nuevamente la indispensable necesidad de la solidaridad latinoamericana para hacer presente en forma efectiva sus intereses, puesto que cada día los países de la denominada "Clase Media" están siendo marginados de la cooperación internacional, en' circunstancia que es desde Amé- rica Latina de donde han nacido las mejores iniciativas que no sólo han denunciado estos abusos sino que han propuesto soluciones.

En quinto lugar, caracteriza la situación mundial lo que yo llamaría una crisis cultural, es decir, una crisis de los valores sobre los cuales se sustentaron los estilos de desarrollo, la concepción del orden social y político y las relaciones internacionales. Cada día se hace más consciente para la humanidad que los problemas de la calidad de vida de las grandes mayorias, los problemas del ser huma- no como persona y sus requerimientos, los problemas de la justicia y de la fraternidad humana, debieran ser realmente el centro y el objetivo del desarrollo. No es una casualidad que en los últimos años las principales Conferencias de las Naciones Unidas hayan sido la de Medio Ambiente, la de Hábitat, la de Población, la de Alimentación, la del Empleo, la de la Mujer, etc. En todas ellas se está reflejando una inquietud moral por el destino del hombre sobre el planeta y por el tipo de relación entre los seres humanos y las obligaciones fundamentales que de ellas derivan para la organización social y económica de los países.

La conciencia moral de la humanidad se ha hecho presente como un fenómeno nuevo en la realidad internacional y está poniendo de manifiesto las injusticias, la importancia de los derechos humanos, en una palabra, la significación profunda del proceso de liberación 
de las grandes mayorías, que significa el esfuerzo constante del hombre por el progreso y no la subordinación de aquél a los grandes intereses económicos, estratégicos o de un progreso sin sentido.

La civilización, como un todo, entra en un profundo proceso de revisión y de cambio. De allí que en las diferentes regiones y países, los principales centros académicos y de investigación e inclusive grandes intereses privados transnacionales empiezan a estudiar y proyectar las tendencias del mundo del faturo, de la vida del hombre sobre el planeta, del nuevo amanecer de la humanidad.

Los grandes centros de poder mundial redefinen sus orientaciones y estrategias buscando no sólo enfrentar solidariamente la coyuntura sino más bien establecer las formas de hacer presente su poder e intereses. En otras palabras, en el reordenamiento de la estructura internacional no habrá cabida para países aislados sin influencia económica, científica $y$ tecnológica, y sin. poder político.

\author{
LA INTEGRACIÓN COHO CONDICIÓN BÁSICA \\ DE UN DESARROLLO AUTÓNOMO
}

Todas estas grandes tendencias del desarrollo reciente de la humanidad obligan como nunca a pensar en el tipo de desarrollo que los países andinos y América Latina en general deben seguir en los próximos 25 años, especialmente si busca una presencia nueva y con rasgos propios en el concierto mundial. Cada día estoy más convencido de la necesidad de una reflexión hacia adentro muy profunda de los latinoamericanos, reconocienclo las diferencias entre nuestros países, para descubrir los elementos cualitativos que hagan que nuestro desarrollo surja incorporando nuestra base cultural, nuestros valores y nuestro sentido de la vicla. No podemos repetir en América Latina con un espiritu de imitación sin prestigio ni provecho, los estilos de desarrollo adoptados por los países industrializados, lo cual nos levaría a la recreación de "civilizaciones desaparecidas", en pueblos atrasados.

El fenómeno que define la política internacional actual es la independencia. Es un proceso dinámico que se expande en alas de las comunicaciones, de la tecnología, del flujo financiero, del comercio y de la progresiva aceptación por parte de la humanidad de ciertos valores, de no escasos temores, pero en todo caso de una común conciencia de soliclaridad de la especie humana.

No sin valor simbólico de proyecciones históricas, al celebrarse el 
centenario de los Estados Unidos, en Filadelfia, en presencia de las autoridades internacionales, se celebró una ceremonia especial publicándose una carta sobre la interdependencia. Esta realidad se presenta antes de que el proceso mundial de descolonización política haya terminado, y ciertamente en circunstancias de que una minima e indispensable independencia económica y tecnológica básica es aún una meta lejana para la inmensa mayoría de los países en desarrollo. $\mathrm{La}$ interdependencia no se ha creado como un proceso orgánico logrado por acuerdos de paises soberanos en busca de una convergencia de intereses, de limitación de sus autonomías y de distribución justa del trabajo, de los recursos y de los beneficios. Es el resultado de la expansión de los centros a través de la acumulación y desarrollo de los recursos tecnológicos y de la organización financiera que los ampara. Me refiero a los Estados Unidos, a la comunidad europea y a Japón, principalmente, que no sólo han creado una cerrada interdependencia entre sí en todos los campos, actuando como tres entidades políticas internacionales solidarias, sino que han desbordado sus fronteras hacia el mundo en desarrollo, no ya en formas de colonialismo tradicional sino que en términos de influencias de hecho en sus requerimientos de materias primas, de mercados, de mano de obra barata. Esta interdependencia tiende a acentuarse también a medio camino entre la confrontación política y militar y la solidaridad económica y tecnológica, entre los países capitalistas y los socialistas desarrollados. Nunca debe olvidarse que la solidaridad es una virtud horizontal y no vertical. El gran desafío para los países de la periferia es que estos se ven obligados a incorporarse a esta interdependencia en el estado en que se encuentran. Algunos de éstos por su espacio y su política diligente, logran insertarse en esta interdependencia obteniendo para sí mayores beneficios que otros, de menor tamaño, menor nivel de desarrollo o menor capacidad de percibir el valor de la autonomía relativa de las opciones, que sólo logran mayores niveles de dependencia.

América Latina tiene en este proceso ventajas y desventajas. Las primeras provienen de que muchos de sus países han alcanzado respetables capacidades tecnológicas y mercados internos que pueden sostener esfuerzos creativos o de adaptación. Por otra parte, la pertenencia al mundo occidental, con valores comunes con los centros que ejercen las influencias directas, otorga una capacidad de negociación que les permitiria aspirar a formar parte de los que obtienen beneficios.

Europa y Estados Uniclos, "cuentan con América Latina". Están acostumbrados a considerar que somos de su mundo. Buen provecho Lan obtenido de esa convicción que normalmente todos compartimos 
pero sobre la cual pocas veces reflexionamos para discriminar entre lo que hay de común y lo que hay de antagónico. Ello debería facilitar la comprensión politica y crear solidaridad creativa. Pero las desventajas son también evidentes. América Latina la estado forzada a realizar simultáneamente, después de la última guerra, la revolución industrial, comenzando por la siderúrgica básica, está sometida al impacto de la revolución tecnológica y es solicitada por el modelo de la sociedad de consumo de los centros. Realizar simultáneamente todo este esfuerzo, expandido a todos los niveles de la población, tiende a sacudir estructuras sociales tradicionales, obliga a traer capitales con tecnologías para exportar bienes de consumo aptos para los centros externos de :altos ingresos y no para las mayorías pobres de los países mismos, hace prescindir cle la investigación propia, crea inflación y coloca a los estados en serias dificultades para satisfacer las necesidades básicas que debe cubrir.

En definitiva, se ha creado en tantas partes un dualismo social que repite al interior de los países el mismo equilibrio que existe a nivel internacional. Por cierto, en estas condiciones las estructuras políticas tradicionales basadas en la expresión libre de las mayorías crujen cuando no se desploman.

Un aspecto que para Ios paises andinos y para la región ha surgido más claramente como factor negativo en este contexto mundial, es el modo de inserción de Anérica Latina en la economía internacional y los efectos que para ello ha tenido la recesión de los países desarrollados, lo que se ha manifestado, no sólo por su impacto negativo sobre la distribución del ingreso al interior de los países, sino también por el decreciente grado de autonomía en aspectos esenciales. América Latina, en el actual nivel de su desarrollo, es profundamente dependiente $\gamma$ vulnerable, tanto por. la alta incidencia que tienen sus exportaciones de minerales o determinados productos agricolas con sus economías, como por el alto nivel de sus importaciones de insumos y de tecnología necesarios para alimentar el proceso de desarrollo industrial emprendido.

Estudios recientes muestran que el crecimiento del producto en la región ha sido acompañado por un más que proporcional aumento de las importaciones. Unido estel hecho a la caída en el valor de las exportaciones y al creciente endeudamiento externo, hacen sumamente difícil que los paises puedan continuar financiando la industrialización de la región a la veź que atender los dilíciles problemas de la inflación, la desocupación creciente y el deterioro de la calidad de la vida.

La recesión afecta más a América Latina que a otras regiones. El producto, que en promedio creció en los últimos años a razón d'e un 
$6 \%$, ha caído al $4 \%$, y la desocupación así como la inflación han aumentado. Si en los años posteriores a la crisis de los años 30 América Latina respondió con un gran esfuerzo de industrialización, ésta, cuy'o costo social y de postergación del sector agricola son bien conocidos, ha liegado a ser insuficiente incluso para absorber el aumento de la población. Por ello es que la crisis actual presenta una nueva oportunidad de reiniciar un crecimiento hacia adentro de la región, más ordenado, con mayor integración de todos los factores productivos y sociales.

¿Cuáles serían algunos de los elementos más definitorios de un nuevo modelo de desarrollo más autónomo para los países andinos y para América Latina como región?

La noción de autonomía la entendemos aqui como la posibilidad de que nuestros países realicen un desarrollo en que el fundamental factor de dinamismo $y$ de control sobre el proceso se encuentre al interior de la región y no fuera de ella. Para lograr este objetivo creo que hay por lo menos cuatro condiciones básicas de las cuales, en gran medida, dependerá en el futuro el papel que la región pueda jugar en el mundo.

En primer término, un desarrollo autonómo requiere un complejo económico especial en el cual exista un alto grado de autosuficiencia de los más indispensables recursos naturales, especialmente los no renovables, que garantice un suministro de materias primas, de energía y de productos alimenticios que le den a ese complejo una relativa independencia del resto de los países y regiones. Este complejo económico-espacial se da sólo a nivel regional, para lo cual, como ya lo hemos dicho, la consolidación de los esquemas subregionales son, una condición sine qua non.

Sólo al observar la región como un todo, sus recursos humanos, sus recursos naturales, su complementariedad agropecuaria, sus recursos de energía, de minerales, etc., puede encontrarse en términos reales el complejo económico espacial que logre evitar en el ámbito mundial y que pueda tener el dinamismo necesario para acelerar el desarrollo de los países.

En segundo término, cada día se hace más evidente que el logro de un modelo o estilo de desarrollo autónomo depende del nivel de creación científica y tecnológica. La región no podrá resolver sus antiguos $y$ nuevos problemas si no realiza un esfuerzo considerable en la ejecución de programas de investigación cientifica, especialmente en aquellos sectores o problemas más importantes para su desarrollo. Todos sabemos que en este sentido, América Latina no sólo no está invirtiendo lo necesario, sino que está lejos de realizar un programa cooperativo de a'to alcance que sirva para crear una 
red especializada de centros de excelencia científico-tecnológica latinoamericana y de empresas mixtas, multinacionales. La brecha en este campo se abre cada día más entre la gran mayoría de los países y el mundo desarrollado. Hablar de autonomía sin capacidad científica y tecnológica es un mito. Pero para lograr esta capacidad debemos realmente aceptar que es imposible hacer ciencia y tecnología a nivel de cada país. Los diferentes "centros de integracion" debieran tener sus propios planes en este campo, los cuales tendrían que irse relacionando con los institutos de otros centros, de tal modo que la región, a fines de siglo, posea una red de creación científica y tecnológica de nivel adecuado. No debemos olvidar que en este campo si quisiéramos producir un impacto y tener, a fines de siglo una real capacidad adecuada a nuestra realidad latinoamericana, debiéramos ya haber comenzado por formular nuestras necesidades reales futuras de ciencia y tecnologia, lo cual implicaria prever y destinar una inversión considerable de recursos financieros $y$ de capacidades humanas. La planificación de los recursos humanos en el campo científico y tecnológico aparece como el de mayor urgencia entre las políticas para derrotar la miseria, modernizar la economía agrícola, reformar la relación. campo-ciudad, crear tecnología, aumentar el nivel de autonomía en el tema de decisiones y aprovechar la capacidad instalada sobre todo en las empresas públicas.

En este sentido, como ya lo he señalado, las realizaciones y programas del Grupo: Andino son un primer paso que debiera seguirse desarrollando y seguramente coordinarse con otros esfuerzos existentes en el área.

Un tercer elemento que aparece como fundamental para cambiar la tendencia a la dependencia que se observa crecientemente en la región, es el mercado ampliado que como ya he dicho es fundamental para un programa de desarrollo acelerado, eficiente y complementario, especialmente en el sector industrial y en el campo de la ciencia y la tecnología.

Es cada clía más evidente que producir con eficiencia implica escala y especialización. Pero, en el mundo del futuro ello será aún más importante. Los grandes paises o agrupaciones de paises con vastos conglomerados humanos y con. un mercado suficiente para producir con eficiencia, serán quienes realmente puedan tener un desarrollo más acelerado y un poder en el nuevo orden mundial. En América Latina, fuera de Brasil y posiblemente Mréxico, es difícil pensar que otros países aislados a fines cle siglo logren una significación económica internacional.

Un cuarto factor determinante para lograr en el futuro un grado 
de mayor autonomia en el desarrollo de nuestros países es la integración y el consenso social al interior de nuestras propias naciones, que surja, realmente de un alto nivel de participación activa de los diversos grupos sociales, tanto en la creación del desarrollo como en los frutos del mismo.

Esta condición, creo que se explica por si misma. Pero siendo obvia, es a mi entender una de las principales causas de nuestros problemas y de que en definitiva los procesos de integración no se consoliden y nuestro esquema de desarrollo no logre persistencia y estabilidad. El mundo del futuro es de muchos riesgos y dificultades. Sólo un fuerte consenso social posibilitará a América Latina proyectarse hacia afuera con una imagen positiva y de respetabilidad. Para ello, creo que un nuevo estilo de desarrollo autónomo debiera centrar sus esfuerzos socioeconómicos en enfrentar los problemas de las grandes mayorías marginadas, única vía para lograr una integración social interna que dé estabilidad, lo cual en definitiva es una condición para la autonomía de la región en el futuro.

Si examináramos una a una lo que yo llamaria las "condiciones del desarrollo autónomo de la América Latina", todas de alguna manera conducen a la integración. Es decir, no hay viabilidad nacional ni hoy ni menos aún en cl futuro mediano y lejano para los pequeños y medianos países de esta América, inclusive para los que hoy aparecen más viables como son Ios tres mayores.

Algunos sectores de los países medianos de Grupo Andino, en este sentido, corren un gran riesgo al creer que tienen una viabilidad a escala internacional. Ello, si fuera posible, sólo sería en el corto plazo. A la postre, no pasaría de ser una artesanía, cada vez más cara y' vulnerable. Para enfrentar los grandes desafíos del futuro, sobre todo en el momento en que se están generando grandes conglomerados económicos, políticos y sociales, cuando inclusive las empresas transnacionales se agrupan para defenderse e influir en la creación de un nuevo orden mundial, no hay destino para pequeños paises aislados, para soberanías nacionales del siglo pasado. Sólo hay destino para los que han sabido proyectarse solidariamente, con objetivos claros que le permitan autonomía y poder a fin de lograr su cuota de respeto y beneficio en el nuevo orden internacional y para crear en América un estilo de desarrollo en el cual cada ser humano tenga dignidad, tenga bienestar y tenga libertad. Ello será posible en la organización de lo que el Libertador Simón Bolívar llamó: "Una nación de repúblicas". 